\title{
Fabrication of Topologically-Complex 3D Microstructures by Femtosecond Laser Machining and Polymer Molding
}

\author{
Allison Schaap, Yves Bellouard \\ Eindhoven University of Technology, PO Box 513, 5600 MB, Eindhoven, The Netherlands \\ a.schaap@tue.nl,y.bellouard@tue.nl
}

\begin{abstract}
We demonstrate the demolding of topologically complex three-dimensional elastomeric microstructures from a femtosecond laser micromachined glass substrate. Demolding success rates of $>90 \%$ are achieved, which are qualitatively supported by a simple mechanical model. OCIS codes: (220.4000) Microstructure fabrication; (220.4610) Optical fabrication
\end{abstract}

\section{Introduction}

The creation of three-dimensional microscaled features on surfaces has many applications, including the control of surface properties such as adhesion [1] and wettability, as well as for fabricating microfluidic, optofluidic, or micromechanical devices. Mask-based photolithography processes offer limited inherent three-dimensionality, and other methods for creating more complex three-dimensional shapes on the microscale, such as 2-photon polymerization and electron-beam lithography, are generally serial (and thus time-consuming) in nature.

As an alternative fabrication technique, we use a two-step process combining femtosecond laser patterning with a chemical etch. The nonlinear multiphoton absorption process locally modifies (but does not ablate) the material in a micron-scaled laser-affected volume; in this volume, the local etching rate in hydrofluoric acid is increased compared to the pristine material. We use this process to fabricate monolithic glass (fused silica) micromolds with complex three-dimensional surface topology, and demonstrate the replication of the negative structures with polydimethylsiloxane (PDMS). The molding process allows many copies of one machined surface to be produced at a low cost, overcoming the limitations of the serial mold fabrication process.

\section{Fabrication}
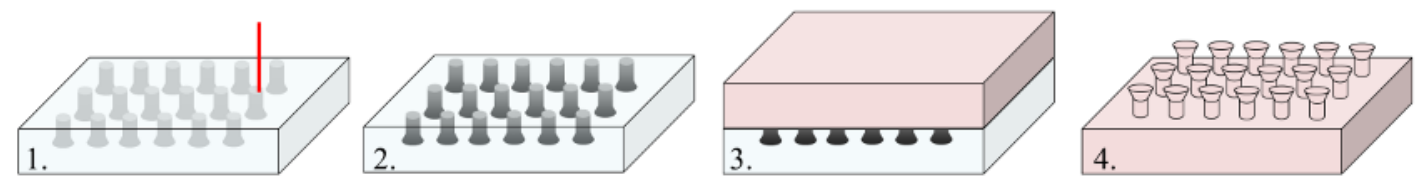

Fig. 1 Fabrication process: a femtosecond laser modifies the substrate (1); the modified regions are selectively etching $2.5 \%$ hydrofluoric acid (2); after the application of a monolayer to decrease mold adhesion (3), PDMS is poured over the glass mold, cured, and removed (5).

An overview of the fabrication process is shown in Fig. 1. Following laser exposure (wavelength $1030 \mathrm{~nm}$; pulse duration $400 \mathrm{fs}$; repetition rate $400 \mathrm{kHz}$; average energy $220 \mathrm{~mW}$; pulse energy of $550 \mathrm{~nJ} /$ pulse), the fused silica sample was etched (2.5\% hydrofluoric acid, room temperature, 9.5 hours) and then coated with a monolayer to decrease the adhesion between the glass and the molding polymer, as described previously [2]. PDMS (Sylgard 184 ) is mixed in a 1:10 ratio of hardener:base, poured on the mold, cured at $65^{\circ} \mathrm{C}$ for 2 hours, and allowed to return to room temperature over several hours. The glass mold and polymer replica are separated by hand.

\section{Mechanical modeling}

To predict the success of the demolding process, we choose the structure shown in Fig $2 \mathrm{a}$ as a representative example. We model the stress at the point of highest deformation, when the narrow-diameter section of the PDMS (section $e$ to $f$, Fig. 3) is stretched around the wider-diameter section of the glass (section $a$ to $b$, Fig. 3). The loading is a combination of the hoop and friction forces resisting the demolding, for which we evaluate the equivalent von Mises stress and compare that to the ultimate tensile strength of PDMS. We take the elastic modulus of PDMS at 1.8 MPa [3], the coefficient of friction of PDMS on glass at 0.3 [4], and the ultimate tensile strength at around $7 \mathrm{MPa}$ [5]. The geometrical values used in the model are based on measurements of the final devices.

\section{Results}

Fig. 2 shows SEM images of some of the demolded structures. The data from the model and the successful demolding rates for this shape are shown in Fig. 3. The structures with d/D<1 (175 structures total) were demolded 
with $>90 \%$ success rate, and the few broken samples were at the extreme of $d / D<0.5$. While the model is only an approximation, it qualitatively aligns with the high demolding success rates we observed.
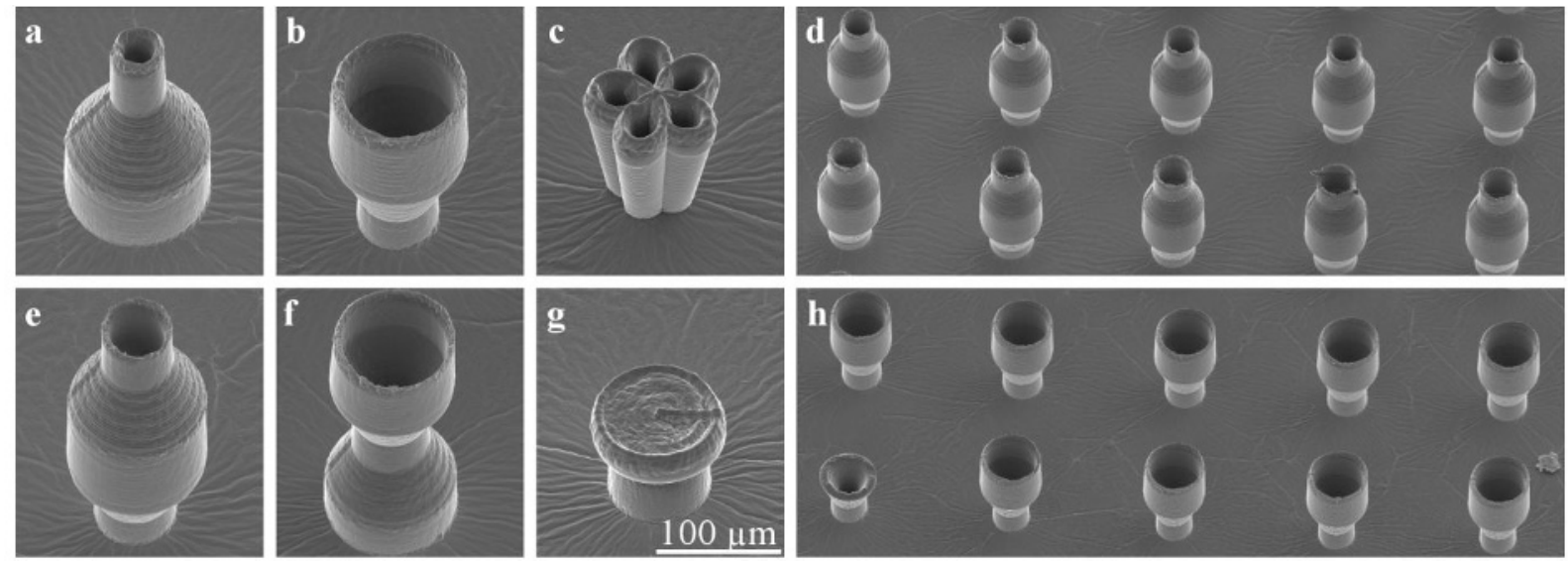

Fig. 2 Examples of demolded structures, including (a, b, e, f) structures where a thin wall of PDMS had to demold around a glass part of larger or smaller diameter; (c) complex thin-walled structures, $(\mathrm{g})$ structures with overhang, and $(\mathrm{d}, \mathrm{h})$ arrays of repeated structures.

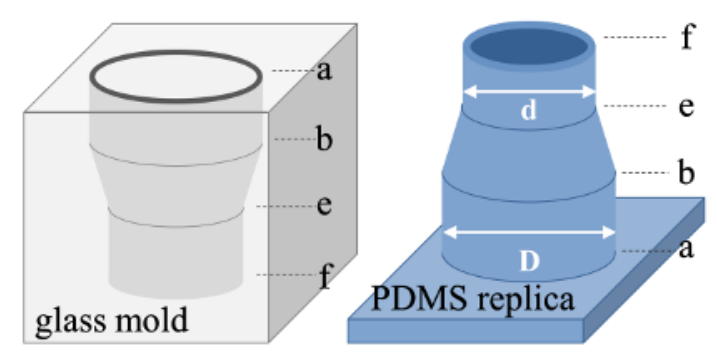

$$
\begin{aligned}
& \text { modelled combined stress } \\
& \text { demolding success rate }
\end{aligned}
$$

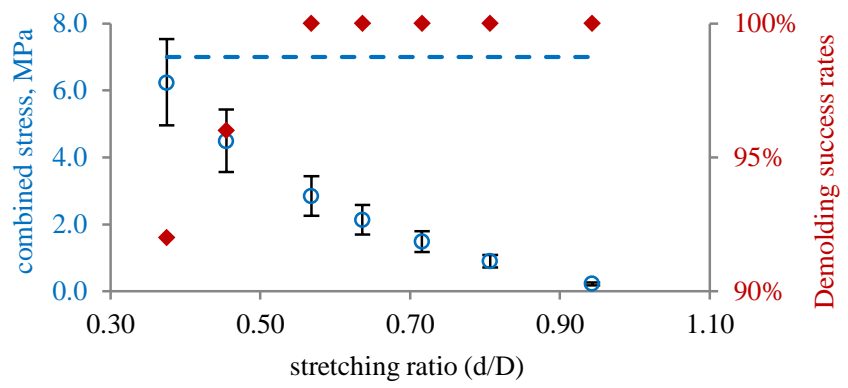

Fig. 3 (Left) The modeled structure: thin-shelled cylinders of varying diameter, with the ratios of $1 / 3 \leq \mathrm{d} / \mathrm{D} \leq 1$. The height of each section is $75 \mu \mathrm{m}$, so the total structure height is $225 \mu \mathrm{m}$. (Right) The combined stress from the model of the structure in Fig. 2a, and the rates of successful demolding, for each ratio of d/D < 1 (i.e., the cases in which the PDMS stretched around the glass during demolding). The PDMS ultimate tensile strength (UTS) is also shown (dashed line). To obtain significant statistics, 25 structures were fabricated at each ratio of d/D. The error bars on the model values represent the effect of changing the coefficient of friction by $\pm 20 \%$.

\section{Conclusion}

The successful demolding of topologically-complex three-dimensional microstructures has been demonstrated. This technique is made possible by non-linear laser-material interactions, which allows the formation of arbitrary threedimensional patterns in glass, creating a monolithic, mechanically robust mold. Structures which require significant stretching of the molding material around the glass are possible with a sufficiently elastic polymer; with PDMS, demolding success rates of over $90 \%$ are achieved, and a simple mechanical model is able to qualitatively predict the demolding success of such structures. This method enables the creation of shapes which are difficult or impossible to achieve with other fabrication techniques, and can produce repeatable, complex molded microstructures with a high yield over an arbitrarily large surface area.

\section{References}

[1] A. Jagota and C.-Y. Hui, "Adhesion, friction, and compliance of bio-mimetic and bio-inspired structured interfaces," Mater. Sci. Eng. R Rep. 72, 253-292 (2011)

[2] F. Madani-Grasset and Y. Bellouard, "Femtosecond laser micromachining of fused silica molds," Opt. Express 18, 21826-21840 (2010).

[3] F. Schneider, et al. , "Mechanical properties of silicones for MEMS," J. Micromech. Microeng. 18, 065008 (2008).

[4] N. S. Tambe and B. Bhushan, "Micro/nanotribological characterization of PDMS and PMMA used for BioMEMS/NEMS applications," Ultramicroscopy 105, 238-247 (2005).

[5] A. Mata, A. J. Fleischman, and S. Roy, "Characterization of polydimethylsiloxane (PDMS) properties for biomedical micro/nanosystems," Biomed Microdevices 7, 281-293 (2005). 